\title{
A Hospital Based Cross Sectional Study on Dietary Status and Associated Factors among People Living with HIV/AIDS in Kigali, Rwanda
}

\author{
Tafadzwa Dzinamarira ${ }^{1,3}$, Gashema Pierre ${ }^{2}$, Elyse Jeanne Umuhire ${ }^{3}$, Michael Habtu ${ }^{1} \&$ Rosemary Okova ${ }^{1}$ \\ ${ }^{1}$ Department of Public Health, Mount Kenya University Rwanda, Kigali, Rwanda \\ ${ }^{2}$ College of Medicine and Health Sciences, University of Rwanda, Kigali, Rwanda \\ ${ }^{3}$ ICAP, Mailman School of Public Health, Kigali, Rwanda \\ Correspondence: Tafadzwa Dzinamarira, Department of Public Health, Mount Kenya University Rwanda, Kigali, \\ Rwanda. E-mail: anthonydzina@gmail.com
}

Received: November 20, 2019

Accepted: December 30, 2019 Online Published: January 9, 2020

doi:10.5539/jfr.v9n1p50

URL: https://doi.org/10.5539/jfr.v9n1p50

\begin{abstract}
Background

Good nutrition empowers PLWH with the ability to fight against infection ultimately slowing down disease progression. Consequently, nutrition management is a crucial component of HIV treatment, care, and support. This study aimed at assessing dietary status and associated factors among PLWH in Kigali, Rwanda.

Methods

We conducted a cross sectional study in three selected hospitals in Kigali from over a six-week period in July August, 2019 to collect data from 204 HIV positive adults enrolled using systematic random sampling. Data was collected using an adapted, validated and pre-tested food frequency questionnaire (FFQ). Descriptive and multiple logistic regression analyses were performed using SPSS version 25 for windows.
\end{abstract}

Results

The proportion of participants with poor dietary status was $15 \%$ based on FFQ responses. The study found only three factors to be independently associated with dietary status. There was an association between dietary status and HIV status disclosure (AOR 2.5; CI $1.25-4.83 ; \mathrm{p}=0.014$ ). There was an association between dietary status and travel time to place of collection of ARVs (AOR 3.2; CI $1.7-5.8 ; \mathrm{p}=0.006$ ). There was an association between dietary status and BMI (AOR 10.2; CI $8.30-16.0$; $<<0.001)$.

Conclusions

Poor dietary status among PLWH remains a concern. The strong association between dietary status and BMI underlines the need for interventions that target PLWH to improve dietary status and ultimately nutrition status

Keywords: dietary status, people living with HIV/AIDS, Rwanda

\section{Introduction}

As of 2016, HIV accounted for more than 1.8 million incidents yearly, with most occurring in resource poor countries (UNAIDS, 2017). Earlier data showed that in 2015, 36.7 million people globally were infected with HIV and AIDS, 1.8 million being children under 15 years (Organization, 2016). In 2015, 1.1 million deaths were reported from HIV with 2.1 million new infections, including 150,000 children (WHO, 2018). It has been reported that close to 70 percent of the burden is in Africa (WHO, 2018), a region with the highest rates of food insecurity. Available evidence shows that PLWH who are undernourished when they start ART are 2-6 times more likely to die within 6 months of ART initiation compared to their normal body mass index counterparts (Munthali, Jacobs, Sitali, Dambe, \& Michelo, 2015). Even on ART, there is a continuous need for PLWH to consume a nutritious diet to maintain weight and prevent micronutrient deficiencies (Audain, Zotor, Amuna, \& Ellahi, 2015). There is also a growing recognition of the role nutritional support within clinical and community services plays in engagement, adherence and retention in care and treatment (Berhe, Tegabu, \& Alemayehu, 2013; Kendall et al., 2014; Tang, Jacobson, Spiegelman, Knox, \& Wanke, 2005). Proper nutrition complements 
the properly adhered ART. Closer to Rwanda, randomized control trials conducted in Kenya and Uganda showed nutritional support to significantly decrease mortality among PLWH initiating ART (PrayGod, Friis, \& Filteau, 2018).

As of a decade ago, more than 800 million people were chronically undernourished (Ivers et al., 2009). The highest burden of both undernutrition and HIV/AIDS has been reported to be in SSA (UNAIDS, 2016). A study conducted in Brazil by Andrade et al. revealed high levels on undernutrition among PLWH at hospitalization with a reported prevalence of $43 \%$ (Andrade et al., 2012). Similar findings were reported in Asia by Hu et al, 2011 with a reported prevalence of malnutrition among PLWH of $37.2 \%$ (Hu et al., 2011). In Senegal, slightly lower prevalence was reported. The prevalence of malnutrition among PLWH, defined by BMI, was $19.2 \%$ in Dakar and 26.3\% in Ziguinchor (Benzekri et al., 2015).

In East Africa, an institution based cross sectional study conducted by Gedle et al., 2015 in Southern Ethiopia reported an overall prevalence of malnutrition was $25.2 \%$ of which 49,19 , and 9 patients were mildly, moderately, and severely malnourished, respectively (Gedle, Gelaw, Muluye, \& Mesele, 2015). Similarly, a multi-center study in Central Ethiopia reported a prevalence of $23.6 \%$ (Gebremichael, Hadush, Kebede, \& Zegeye, 2018). These figures raise concerns as lower dietary diversity has been associated with greater mortality and poor clinical outcomes among PLWH (Palermo, Rawat, Weiser, \& Kadiyala, 2013; Rawat, McCoy, \& Kadiyala, 2013). There is no published literature for dietary status and associated factors among PLWH in Rwanda. The current study aimed to assess dietary status and associated factors among PLWH in Kigali, Rwanda.

\section{Materials and Methods}

\subsection{Study Design}

The study was a cross sectional survey. We adopted this study design given the nature of the research question. To obtain a snapshot of the current proportion of dietary status and associated factors among PLWH in Kigali, Rwanda, such a study design was best to survey the study population and answer the research questions.

\subsection{Study Setting}

Kigali City Province is the Capital City of Rwanda. The province has a total of 42 health facilities spread over the three districts; 21 in Gasabo, 10 in Kicukiro and 11 in Nyarugenge (NISR \& Rwanda, 2014), it has a surface area of $730 \mathrm{~km}^{2}$. The population of Kigali was 1,132,686 as of the 2012 national census (NISR \& Rwanda, 2014). Among adults $15-64$ years old, HIV prevalence in City of Kigali is 4.3\% (ICAP, 2019). One health facility in each district in Kigali Province was purposively selected based on researcher convenience and volumes of ARV clinic attendees.

\subsection{Sampling and Data Collection Instrument}

At each study site, participants were enrolled into the study by using simple random sampling. The questionnaire addressed: socio-demographic characteristics of the interviewee (sex, age, education, religion, marital status, occupation, duration on ART); outcome variable (dietary status). The food frequency questionnaire (FFQ) was adapted from a validation study conducted in the Rwanda context in 2016 (Yanagisawa et al., 2016).

\subsection{Data Quality}

We conducted a pretest of the survey questionnaire on 15 respondents in a non-sampled hospital. Consistency, understandability, and flow of questions was tested and revised accordingly. To ensure high data quality, the interviewers (GP and EJU) received a survey specific training for one-week. TD provided close supervision of the interviewers during data collection and the questionnaires were thoroughly edited to make sure that relevant questions have been responded to and coded according to the code designed for the study.

\subsection{Data Entry and Statistical Analysis}

Data coding and verification of responses were made on the same day and any missing information corrected. The cleaned data were entered into the computer using SPSS version 25 platform. Quantitative data was analyzed using descriptive statistics. The FFQ assessed consumption of energy, protein, iron and vitamin A. Based on the participant responses a mean food frequency assessment score of $49.5 \%$ was calculated. Participants that scored less than the mean were classified as poor dietary status while mean and over were classified as good dietary status. This allowed for the dependent variable to be dichotomous with subsequent analysis. The student t-test or chi-square test and logistic regression were used to determine study variables associated with dietary status. All statistical tests were concluded at $5 \%$ level of significance. 


\subsection{Ethical Considerations}

This study has been ethically reviewed and approved by the University Teaching Hospital of Kigali Ethics Committee (Approval number: EC/CHUK/0129/2019).

\section{Results}

\subsection{Socio-demographic Characteristics}

A total of 204 participants were enrolled with average age of 30.3 and majority (55\%) being female. Of these, $88 \%$ reported to have ever attended school while only $5 \%$ were currently enrolled in school. $61 \%$ were educated up to primary level only while $38 \%$ reported to have worked in the past 12 months. $18 \%$ were married and of these only $3 \%$ reported to have more than one wife/husband. Participant demographic characteristics are presented in Table 1.

Table 1. Demographic characteristics of the PLWH enrolled.

\begin{tabular}{ll}
\hline Variable & Value \\
Age in years, mean (SD) & $30.3(5.3)$ \\
Sex, $\mathrm{n}(\%)$ & \\
Male & $92(45)$ \\
Education level, $\mathrm{n}(\%)$ & \\
$\quad$ Nursery & $4(2)$ \\
$\quad$ Primary & $121(59)$ \\
$\quad$ Secondary & $65(32)$ \\
$\quad$ Higher & $14(7)$ \\
Work in the last 12 months for which you received cash or goods as payment $\mathrm{n}(\%)$ & \\
$\quad$ Yes & $78(38)$ \\
Work in the last seven days for which you received cash or goods as payment $\mathrm{n}(\%)$ & \\
$\quad$ Yes & $49(24)$ \\
Ever been married or lived together with a [man/woman] as if married n $(\%)$ & \\
$\quad$ Yes & $169(83)$ \\
Marital status, $\mathrm{n}(\%)$ & \\
$\quad$ Married & $35(18)$ \\
Living together & $45(22)$ \\
Widowed & $50(24)$ \\
Divorced & $6(3)$ \\
$\quad$ Separated & $35(17)$ \\
Single & $38(16)$ \\
Number of live-in partners $n$ & \\
0 & \\
1 & $124(63)$ \\
2 & $68(35)$ \\
3 & $3(2)$ \\
Number of live-in partners who live elsewhere? $\mathrm{n}(\%)$ & $2(1)$ \\
0 & \\
1 & $130(66)$ \\
2 & $64(32)$ \\
\hline
\end{tabular}

\subsection{Dietary Status}

Of the 204 participants, $15 \%$ had poor dietary status based on their FFQ responses.

\subsection{Factors Associated with Dietary Status}

The study found only three factors to be independently associated with dietary status. There was an association between dietary status and HIV status disclosure (AOR 2.5; CI $1.25-4.83 ; \mathrm{p}=0.014$ ). There was an association between dietary status and travel time to place of collection of ARVs (AOR 3.2; CI 1.7 - 5.8; $\mathrm{p}=0.006$ ). There was an association between dietary status and BMI (AOR 10.2; CI $8.30-16.0 ; \mathrm{p}<0.001$ ). More information is presented on Figure 1. 


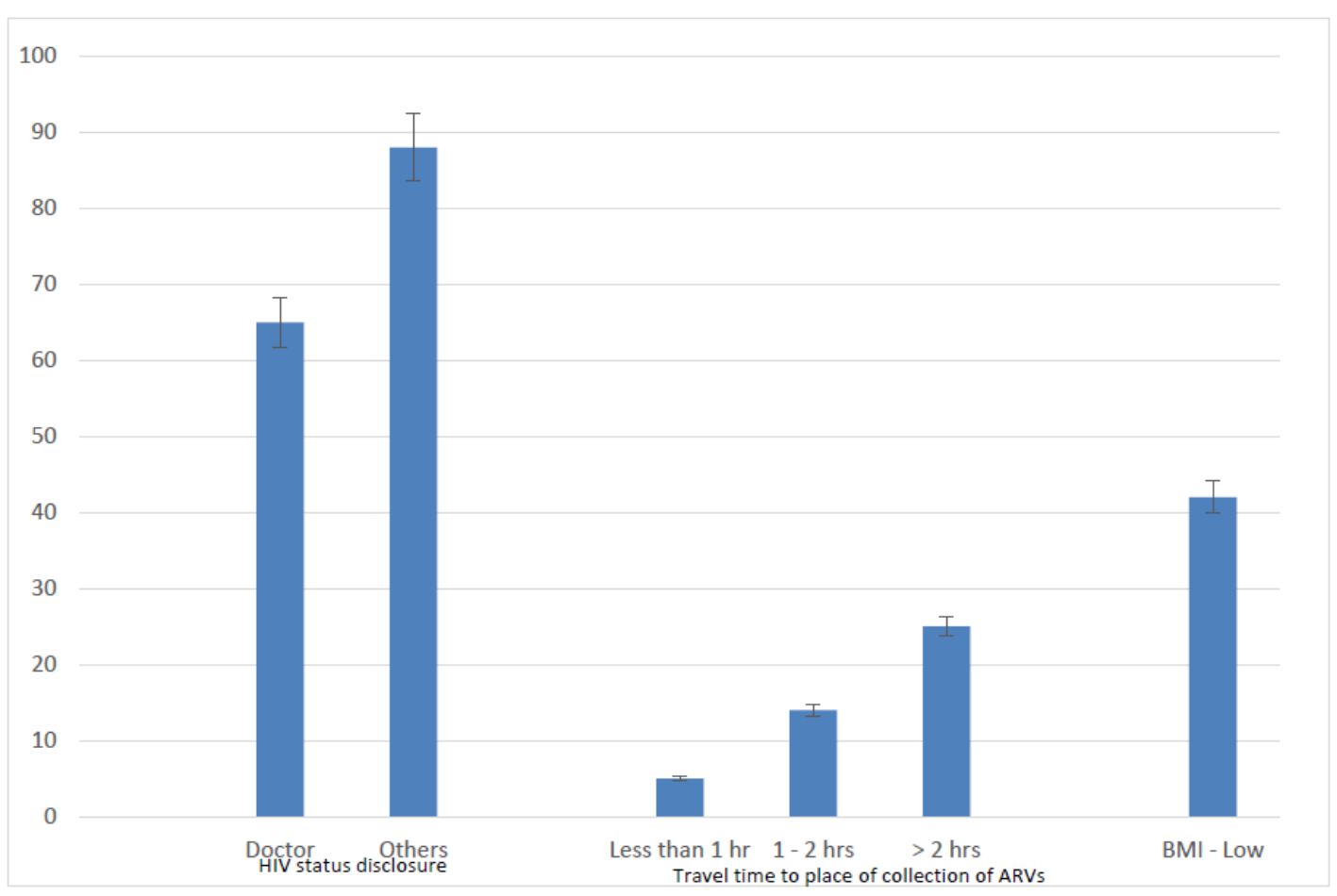

Figure 1. Factors associated with poor dietary status among PLWH in Kigali, Rwanda

\section{Discussion}

The current study revealed $15 \%$ of PLWH in Kigali, Rwanda to have poor dietary status. The findings of the current study mainly noted that some of the main factors, which are associated with poor dietary status include HIV status disclosure, travel time to clinic and BMI. Globally, multi-organizational efforts have been launched and recommendations made by The U.S. President's Emergency Plan for AIDS Relief (PEPFAR), and endorsed by WHO, UNAIDS, and the World Food Program. The nutrition assessment, counseling and support model is known as NACS (Tang, Quick, Chung, \& Wanke, 2015). As of 2017, South Africa, Mozambique and Nigeria were in early planning phases. Cote d'Ivoire, Ghana, Ethiopia, Tanzania, Namibia and Zambia were at program expansion stage. Only Kenya and Malawi were in full implementation at national scale. Findings of the current study underscore the need for nutritional management of PLWH to be prioritized in Rwanda.

In the current study, $15 \%$ of the participants had poor dietary status based on their FFQ responses. A slightly higher prevalence of 25.2\% was reported by Gedle et al. 2015 in Ethiopia (Gedle et al., 2015). However, a study by Hailemariam et al. in Ethiopia established that $12.3 \%$ of PLWH had poor dietary status (Hailemariam, Bune, $\&$ Ayele, 2013). The findings of the study that was done by Gebremichael et al. noted that the prevalence of poor dietary status was $23.6 \%$ (Gebremichael et al., 2018). In Senegal, Benzekri et al. established that $19.2 \%$ in Dakar and 26.3\% in Ziguinchor had poor dietary status (Benzekri et al., 2015). It is worth noting that the prevalence the current study reports is comparable to what has been reported by Argemi et al. who established that $11.2 \%$ adults initiating ART were malnourished (Argemi et al., 2012). The difference between these findings and the current study findings could be attributed to the lower BMI lower limit set by Argemi et al of 16 (Argemi et al., 2012). However, much higher prevalence have been reported elsewhere. Mulu et al. noted a much higher prevalence [46.8\%] in comparison to the findings of the current study (Mulu, Hamza, \& Alemseged, 2016). Hadgu et al. reported a prevalence of $42.3 \%$ (Hadgu, Worku, Tetemke, \& Berhe, 2013). This is way higher than the findings of the current study.

Most of the studies have tried to explore some of the main factors that are associated with poor dietary status among PLWH. For instance, the findings of Gedle et al. revealed that living within the rural areas, anemia, as well as intestinal parasitic co-infection was significantly linked to poor dietary status (Gedle et al., 2015). The study also made the conclusion that the prevalence of malnutrition among PLWH getting ART in Butajira was very high. Similarly, the study, which was carried out by Hailemariam et al. indicated that some of the factors, which were closely linked to poor dietary status among PLWH include unemployment, WHO clinical stage four, gastrointestinal symptoms, as well as past opportunistic infections (Hailemariam et al., 2013). Some of the main 
factors, which have been linked to poor dietary status among the HIV/AIDS patients included unemployment, clinical stages of AIDS progression, CD4 count less than $350 \mathrm{cells} / \mu \mathrm{l}$, tuberculosis, the duration on antiretroviral therapy as well as household food insecurity (Gebremichael et al., 2018). The study by Benzekri et al. noted that severe food insecurity was linked to the missing of clinic appointments and also to the failure of the PLWH to take antiretroviral therapy because of hunger (Benzekri et al., 2015). The findings of the study that was done by Hadgu et al. noted that household food insecurity, inadequate dietary diversity, anemia, as well as the general lack of nutritional support were the major independent predictors of poor dietary status (Hadgu et al., 2013). It can be noted that most of the previous studies have given different factors to be associated with dietary status.

\section{Conclusion}

Based on the findings of the current study, poor dietary status among PLWH remains a concern. The strong association between dietary status and BMI underlines the need for interventions that target PLWH to improve dietary status and ultimately nutrition status. Larger studies with designs of more scientific rigour evaluating dietary status and associated factors may help shed more light into the research problem. This study has presented baseline findings which may be used to guide future research.

\section{Limitations}

Cross sectional studies by nature are not able to demonstrate causality. Further, the findings may not be generalized for the entire population. Additionally, the data collection tool to be used for dietary assessment collected descriptive qualitative information only. However, it is anticipated the tool yielded useful findings as the tool used was adapted from a Rwanda validated food FFQ with guidelines from WHO. Finally, the research did not include biomarkers, the gold standard for any nutritional assessment to validate the self-reported dietary status.

\section{Declarations}

\section{Acknowledgements}

We thank all the participants who took time to complete the questionnaires for this study.

\section{Competing Interests}

The authors declare that they have no competing interests, which may have inappropriately influenced them in writing this article.

\section{Funding}

N/A

\section{Availability of Data and Materials}

All data generated or analysed in this study is available from the corresponding author following a written request with permission from University Teaching Hospital of Kigali Ethics Committee.

\section{References}

Andrade, C. S., Jesus, R. P., Andrade, T. B., Oliveira, N. S., Nabity, S. A., \& Ribeiro, G. S. J. P. O. (2012). Prevalence and characteristics associated with malnutrition at hospitalization among patients with acquired immunodeficiency syndrome in Brazil. PLOS ONE, 7(11), e48717. https://doi.org/10.1371/journal.pone.0048717

Argemi, X., Dara, S., You, S., Mattei, J. F., Courpotin, C., Simon, B., ... Lefebvre, N. J. A. (2012). Impact of malnutrition and social determinants on survival of HIV-infected adults starting antiretroviral therapy in resource-limited settings. AIDS, 26(9), 1161-1166. https://doi.org/10.1097/QAD.0b013e328353f363

Audain, K. A., Zotor, F. B., Amuna, P., \& Ellahi, B. (2015). Food supplementation among HIV-infected adults in Sub-Saharan Africa: impact on treatment adherence and weight gain. Proceedings of the Nutrition Society, 74(4), 517-525. https://doi.org/10.1017/S0029665115000063

Benzekri, N. A., Sambou, J., Diaw, B., Sall, F., Niang, A., Ba, S., ... Seydi, M. J. P. O. (2015). High prevalence of severe food insecurity and malnutrition among HIV-infected adults in Senegal, West Africa. PLOS ONE, 10(11), e0141819. https://doi.org/10.1371/journal.pone.0141819

Berhe, N., Tegabu, D., \& Alemayehu, M. J. B. i. d. (2013). Effect of nutritional factors on adherence to antiretroviral therapy among HIV-infected adults: a case control study in Northern Ethiopia. BMC Infectious Diseases, 13(1), 233. https://doi.org/10.1186/1471-2334-13-233

Gebremichael, D. Y., Hadush, K. T., Kebede, E. M., \& Zegeye, R. T. (2018). Food Insecurity, Nutritional Status, 
and Factors Associated with Malnutrition among People Living with HIV/AIDS Attending Antiretroviral Therapy at Public Health Facilities in West Shewa Zone, Central Ethiopia. BioMed Research International, 2018. https://doi.org/10.1155/2018/1913534

Gedle, D., Gelaw, B., Muluye, D., \& Mesele, M. (2015). Prevalence of malnutrition and its associated factors among adult people living with HIV/AIDS receiving anti-retroviral therapy at Butajira Hospital, southern Ethiopia. BMC Nutrition, 1(1), 5. https://doi.org/10.1186/2055-0928-1-5

Hadgu, T. H., Worku, W., Tetemke, D., \& Berhe, H. J. (2013). Undernutrition among HIV positive women in Humera hospital, Tigray, Ethiopia, 2013: antiretroviral therapy alone is not enough, cross sectional study. BMC Public Health, 13(1), 943. https://doi.org/10.1186/1471-2458-13-943

Hailemariam, S., Bune, G. T., \& Ayele, H. T. J. (2013). Malnutrition: Prevalence and its associated factors in People living with HIV/AIDS, in Dilla University Referral Hospital. Archives of Public Health, 71(1), 13. https://doi.org/10.1186/0778-7367-71-13

Hu, W., Jiang, H., Chen, W., He, S.-H., Deng, B., Wang, W.-Y., ... Zeng, J. J. (2011). Malnutrition in hospitalized people living with HIV/AIDS: evidence from a cross-sectional study from Chengdu, China. Asia Pacific Journal of Clinical Nutrition, 20(4), 544.

ICAP. (2019). Summary Sheet: Preliminary Findings: Rwanda population-based HIV Impact Assessment RPHIA 2018-2019.

Ivers, L. C., Cullen, K. A., Freedberg, K. A., Block, S., Coates, J., Webb, P., \& Mayer, K. (2009). HIV/AIDS, undernutrition, and food insecurity. Clinical Infectious Diseases, 49(7), 1096-1102. https://doi.org/10.1086/605573

Kendall, T., Danel, I., Cooper, D., Dilmitis, S., Kaida, A., Kourtis, A. P., ... Moran, A. C. (2014). Eliminating preventable HIV-related maternal mortality in sub-Saharan Africa: what do we need to know?. JAIDS Journal of Acquired Immune Deficiency Syndromes, 67(Suppl 4), S250. https://doi.org/10.1097/QAI.0000000000000377

Mulu, H., Hamza, L., \& Alemseged, F. (2016). Prevalence of malnutrition and associated factors among hospitalized patients with acquired immunodeficiency syndrome in Jimma University Specialized Hospital, Ethiopia. Ethiopian Journal of Health Sciences, 26(3), 217-226. https://doi.org/10.4314/ejhs.v26i3.4

Munthali, T., Jacobs, C., Sitali, L., Dambe, R., \& Michelo, C. J. (2015). Mortality and morbidity patterns in under-five children with severe acute malnutrition (SAM) in Zambia: a five-year retrospective review of hospital-based records (2009-2013). Archives of Public Health, 73(1), 23. https://doi.org/10.1186/s13690-015-0072-1

Nisr, M. J. T. R. O. P. S., Structure, \& Rwanda, D. N. I. O. S. O. (2014). Rwanda fourth population and housing census 2012.

Organization, W. H. (2016). Progress report 2016: prevent HIV, test and treat all: WHO support for country impact.

Palermo, T., Rawat, R., Weiser, S. D., \& Kadiyala, S. (2013). Food access and diet quality are associated with quality of life outcomes among HIV-infected individuals in Uganda. PLOS ONE, 8(4), e62353. https://doi.org/10.1371/journal.pone.0062353

PrayGod, G., Friis, H., \& Filteau, S. J. T. 1. H. (2018). Nutritional support to reduce mortality in patients with HIV?. The Lancet, 5(5), e202-e204. https://doi.org/10.1016/S2352-3018(18)30047-X

Rawat, R., McCoy, S. I., \& Kadiyala, S. (2013). Poor diet quality is associated with low CD4 count and anemia and predicts mortality among antiretroviral therapy-naive HIV-positive adults in Uganda. JAIDS Journal of Acquired Immune Deficiency Syndromes, 62(2), 246-253.

https://doi.org/10.1097/QAI.0b013e3182797363

Tang, A. M., Jacobson, D. L., Spiegelman, D., Knox, T. A., \& Wanke, C. (2005). Increasing risk of 5\% or greater unintentional weight loss in a cohort of HIV-infected patients, 1995 to 2003. JAIDS Journal of Acquired Immune Deficiency Syndromes, 40(1), 70-76. https://doi.org/10.1097/01.qai.0000159627.54149.2e

Tang, A. M., Quick, T., Chung, M., \& Wanke, C. (2015). Nutrition Assessment, Counseling, and Support (NACS) interventions to improve health-related outcomes in people living with HIV/AIDS: a systematic review of the literature. JAIDS Journal of Acquired Immune Deficiency Syndromes, 68(3), S340. https://doi.org/10.1097/QAI.0000000000000521 
UNAIDS. (2016). AIDS by the numbers. Retrieved from Geneva, Switzerland:

UNAIDS. (2017). Ending AIDS: Progress towards the 90-90-90 targets. Retrieved from Geneva, Switzerland:

WHO. (2018). HIV Self-Testing Strategic Framework: A Guide For Planning, Introducing And Scaling Up HIV Testing Services. Retrieved from https://apps.who.int/iris/bitstream/handle/10665/275521/9789241514859-eng.pdf?ua=1

Yanagisawa, A., Sudo, N., Amitani, Y., Caballero, Y., Sekiyama, M., Mukamugema, C., ... Hirotaka, M. (2016). Development and Validation of a Data-Based Food Frequency Questionnaire for Adults in Eastern Rural Area of Rwanda. Nutrition and Metabolic Insights, 9, NMI. S38374. https://doi.org/10.4137/NMI.S38374

\section{Copyrights}

Copyright for this article is retained by the author(s), with first publication rights granted to the journal.

This is an open-access article distributed under the terms and conditions of the Creative Commons Attribution license (http://creativecommons.org/licenses/by/4.0/). 